\title{
IMPROVED SYNTHESIS OF FARINOMALEIN AND ITS ANALOGUES
}

\author{
Sachin T. Aiwale, *a Paola Sardi, ${ }^{\text {b }}$ Sabrina Dallavalle ${ }^{a}$ \\ ${ }^{a}$ Dipartimento di Scienze Molecolari Agroalimentari, Università degli Studi di Milano, via Celoria \\ 2, 20133 Milano, Italy \\ ${ }^{b}$ Dipartimento di Protezione dei Sistemi Agroalimentare e Urbano e Valorizzazione delle \\ Biodiversità, Università degli Studi di Milano, Via Celoria 2, 20133 Milano, Italy
}

A practical and convenient synthesis of the fungicidal natural compound Farinomalein is described starting from readily available ethyl 3-methyl-2-oxobutyrate and triethyl phosphonoacetate, employing a Horner-Wadsworth-Emmons condensation as the key step.

Keywords: Natural compound; Antifungal; Synthesis; Farinomalein analogues

\section{INTRODUCTION}

Farinomalein $\mathbf{1}$ is a relatively structurally simple maleimide isolated in 2009 from the entomopathogenic fungus Paecilomyces farinosus, by Nihira et.al.. ${ }^{[1]}$. Farinomalein shows potent inhibition of Phytophthora sojae, a plant pathogen that causes every year enormous damage to Soybean crops. ${ }^{[2]}$.

Address correspondence to Sachin T. Aiwale. Tel +39-2-50316818; FAX +39-2-50316801. email:sachin.aiwale@unimi.it 
Intrigued by the interesting biological activity and due to our ongoing interest in the synthesis of antifungal natural products, we developed a practical synthesis of Farinomalein, which may, in principle, have value in the large scale preparation of the natural compound itself as well as of various analogues.

Recently, a three-step synthesis of Farinomalein has been reported by Miles and Yan. ${ }^{[3]}$ The sequence is based on the reaction of isovaleraldehyde and glyoxylic acid to give a $\gamma$ hydroxybutenolide. Successive oxidation to the corresponding anhydride and treatment with $\square \beta$ alanine affords Farinomalein. However, the Authors themselves state that their synthesis is difficult to scale-up ${ }^{[3]}$, due to the use of hazardous and expensive oxidant Dess-Martin periodinane. Herein we report a different synthetic approach to Farinomalein, developed in four steps with high yield and without using any hazardous chemical. A Horner-Wadsworth-Emmons condensation is the key step of the synthetic pathway.

\section{RESULTS AND DISCUSSION}

As outlined in Scheme 1, commercially available ethyl 3-methyl-2-oxobutyrate 2 was reacted with triethylphosphonoacetate 3 in the Horner-Wadsworth-Emmons conditions ${ }^{[4]}$ to give the diester $\mathbf{4}^{[5]}$ in $80 \%$ yield as a faint yellow oil. Hydrolysis of the diester using $\mathrm{LiOH}$ gave in $96 \%$ yield the cisdiacid $\mathbf{5}^{[6]}$, that was then stirred with TFAA at room temperature to obtain the anhydride $\mathbf{6}^{[7]}$ in quantitative yield. Finally, the treatment of the anhydride with $\beta$-alanine in acetic acid. ${ }^{[4,8]}$ provided Farinomalein 1 in $60 \%$ yield. The procedure was scaled up to $10 \mathrm{~g}$ batches.

\section{Scheme 1}

The methodology developed is flexible and allows ready preparation of different analogues, to support future structure-activity relationship studies. 
A first series of Farinomalein analogues was prepared as depicted in Scheme 2. The common intermediate 6 was reacted with different commercially available aminoacids such as $L$-leucine, $L$ phenylalanine, $L$-valine and glycine to obtain analogues with different alkyl chains on the maleimide skeleton.

\section{Scheme 2}

As the activity of Farinomalein against Phytophthora sojae is already documented in the literature, we decided to assess the activity of $\mathbf{1}$ and analogues against Cladosporium cladosporioides, a fungal plant pathogen that affects wheat, using the method of bioautography. ${ }^{[9-10]}$ (Table 1). The bioassay results confirmed that Farinomalein is endowed with antifungal activity also on this and likely on other plant pathogens, the minimum amount required for the inhibition of fungal growth on thin-layer chromatography plates being $5 \mu \mathrm{g}$. Moreover, the results clearly indicate that the introduction of different chains was detrimental for activity. In fact, the minimum amount of compounds 7a-d required for fungal growth inhibition was 6-10 fold higher than for 1 (30-50 $\mu \mathrm{g})$.

Table 1. Antifunal activity of Farinomalein and analogues

\begin{tabular}{|c|c|}
\hline Compound & $\begin{array}{l}\text { Antifungal activity }(\mu \mathrm{g})^{\mathrm{a}} \\
\text { C. cladosporioides }\end{array}$ \\
\hline $\mathbf{1}$ & 5 \\
\hline $\mathbf{7 a}$ & 50 \\
\hline $\mathbf{7 b}$ & 50 \\
\hline $\mathbf{7 c}$ & 30 \\
\hline $\mathbf{7 d}$ & 50 \\
\hline prochloraz & 0.1 \\
\hline
\end{tabular}


${ }^{a}$ Minimum amount required for the inhibition

of fungal growth on thin-layer chromatography

plates (TLC)

\section{EXPERIMENTAL}

\section{General methods}

All reagents and solvents were reagent grade or were purified by standard methods before use. Melting points were determined in open capillaries. NMR spectra were recorded with a Bruker AMX 300 spectrometer. The Elemental Analyses were recorded with a CARLO ERBA EA 1108 instrument. Solvents were routinely distilled prior to use; anhydrous tetrahydrofuran (THF) and ether $\left(\mathrm{Et}_{2} \mathrm{O}\right)$ were obtained by distillation from sodium-benzophenone ketyl; dry dichloromethane was obtained by distillation from phosphorus pentoxide. All reactions requiring anhydrous conditions were performed under a positive nitrogen flow, and all glassware were oven dried and/or flame dried. Isolation and purification of the compounds were performed by flash column chromatography on silica gel 60 (230-400 mesh). Analytical thin-layer chromatography (TLC) was conducted on TLC plates (silica gel $60 \mathrm{~F}_{254}$, aluminum foil) visualized by UV light and spraying with phosphomolybdic acid and $p$-anisaldehyde.

3-(3-Isopropyl-2.5-dihydro-pyrrol-1-yl)-propionic acid (1). To the stirred solution of 6 (0.100g $7.1 \mathrm{mmol})$ in $\mathrm{AcOH}(0.5 \mathrm{ml}) \beta$-Alanine $(0.063 \mathrm{~g}, 7.1 \mathrm{mmol})$ was added at room temperature. The reaction mixture was refluxed for $2 \mathrm{~h}$, then cooled to $60^{\circ} \mathrm{C}$ and the solvent was evaporated under vacuum. To the crude product EtOAc $(10 \mathrm{ml})$ was added and the organic layer was washed with $2 \mathrm{~N}$ $\mathrm{HCl}(5 \mathrm{ml})$ and brine, then dried over $\mathrm{Na}_{2} \mathrm{SO}_{4}$ and concentrated. Purification by flash column chromatography afforded $\mathbf{1}$ as a white solid $(0.089 \mathrm{~g} 60 \%)$. M.p. $76^{\circ} \mathrm{C}, \mathrm{Rf}=0.36$ (diethyl 
ether/hexane 50:50). ${ }^{1} \mathrm{HMR}\left(300 \mathrm{MHz}, \mathrm{CDCl}_{3}\right):{ }^{1} \mathrm{H} \mathrm{NMR}\left(300 \mathrm{MHz}, \mathrm{CDCl}_{3}\right) \delta: 6.25(1 \mathrm{H}, \mathrm{d}, \mathrm{J}=1.8$ Hz), $3.80(2 \mathrm{H}, \mathrm{t}, \mathrm{J}=7.0 \mathrm{~Hz}), 2.80-2.95(1 \mathrm{H}, \mathrm{m}), 2.70(2 \mathrm{H}, \mathrm{t}, \mathrm{J}=7.0 \mathrm{~Hz}), 1.21(6 \mathrm{H}, \mathrm{d}, \mathrm{J}=6.9 \mathrm{~Hz})$. ${ }^{1} \mathrm{H}$ and ${ }^{13} \mathrm{C}$ NMR $\left(\mathrm{CD}_{3} \mathrm{OD}\right)$ completely matched those reported in the isolation paper. ${ }^{[1]}$ Anal. Calcd for $\mathrm{C}_{10} \mathrm{H}_{13} \mathrm{NO}_{4}$ : C 56.86; H 6.20; N 6.63. Found: C 56.98; H 6.10; N 6.51.

The procedure described above was used to prepare compounds 7a-d.

(3-Isopropyl-2,5-dioxo-2,5-dihydropyrrol-1-yl)acetic acid (7a). Oil. Yield 57\% Rf $=0.4$ (diethyl ether/hexane 50:50). ${ }^{1} \mathrm{H}$ NMR (300 MHz, $\left.\mathrm{CDCl}_{3}\right): \delta: 7.80(1 \mathrm{H}, \mathrm{brs}), 6.30(1 \mathrm{H}, \mathrm{s}), 4.28(2 \mathrm{H}, \mathrm{s})$, 2.75-2.95 (1H, m), $1.20(6 \mathrm{H}, \mathrm{d}, \mathrm{J}=7.0 \mathrm{~Hz}) ;{ }^{13} \mathrm{C} \mathrm{NMR}\left(75 \mathrm{MHz}, \mathrm{CDCl}_{3}\right): \delta: 172.5,170.3,169.9$, 156.5, 125.0, 38.3, 26.0, 20.8. Anal. Calcd for $\mathrm{C}_{9} \mathrm{H}_{11} \mathrm{NO}_{4}$ : C 54.82; H 5.62; N 7.10. Found: C 55.01; H 5.41; N 7.01.

2-(3-Isopropyl-2,5-dioxo-2,5-dihydropyrrol-1-yl)-3-methylbutyric acid (7b) Oil. Yield 41\% Rf $=0.5$ (diethyl ether/hexane 50:50). ${ }^{1} \mathrm{H}$ NMR (300 MHz, $\left.\mathrm{CDCl}_{3}\right): \delta: 9.00(1 \mathrm{H}, \mathrm{brs}), 6.25(1 \mathrm{H}, \mathrm{s})$, $4.40(1 \mathrm{H}, \mathrm{d}, \mathrm{J}=8.4 \mathrm{~Hz}), 2.80-2.95(1 \mathrm{H}, \mathrm{m}), 2.60-2.75(1 \mathrm{H}, \mathrm{m}), 1.20(6 \mathrm{H}, \mathrm{d}, \mathrm{J}=7.0 \mathrm{~Hz}), 1.10(3 \mathrm{H}$, $\mathrm{d}, \mathrm{J}=7.0 \mathrm{~Hz}), 0.85(3 \mathrm{H}, \mathrm{d}, \mathrm{J}=7.0 \mathrm{~Hz}) .{ }^{13} \mathrm{C} \mathrm{NMR}\left(75 \mathrm{MHz}, \mathrm{CDCl}_{3}\right): \delta: 174.3,170.6,170.3,156.0$, 124.7, 57.6, 28.4, 25.9, 20.8 (x3), 19.5. Anal. Calcd for $\mathrm{C}_{12} \mathrm{H}_{17} \mathrm{NO}_{4}$ : C 60.24; H 7.16; N 5.85. Found: C 60.66; H 7.00; N 5.57.

2-(3-Isopropyl-2,5-dioxo-2,5-dihydropyrrol-1-yl)-4-methylpentanoic acid (7c) Oil. Yield 53\% $\mathrm{Rf}=0.3$ (diethyl ether/hexane 50:50). ${ }^{1} \mathrm{H}$ NMR $\left(300 \mathrm{MHz}, \mathrm{CDCl}_{3}\right) \delta: 6.28(1 \mathrm{H}, \mathrm{s}), 4.75(1 \mathrm{H}, \mathrm{dd}, \mathrm{J}$ $=4.6,11.3 \mathrm{~Hz}), 2.80-2.95(1 \mathrm{H}, \mathrm{m}), 2.20-2.32(1 \mathrm{H}, \mathrm{m}), 1.80-1.95(1 \mathrm{H}, \mathrm{m}), 1.35-1.50(1 \mathrm{H}, \mathrm{m}), 1.20$ $(6 \mathrm{H}, \mathrm{d}, \mathrm{J}=7.0 \mathrm{~Hz}), 0.90(6 \mathrm{H}, \mathrm{d}, \mathrm{J}=7.0 \mathrm{~Hz}) .{ }^{13} \mathrm{C} \mathrm{NMR}\left(75 \mathrm{MHz}, \mathrm{CDCl}_{3}\right): \delta: 175.9,170.6,170.3$, 
156.1, 124.9, 50.3, 37.0, 25.9, 25.2, 23.1, 21.0, 20.8 (x 2). Anal. Calcd for $\mathrm{C}_{13} \mathrm{H}_{19} \mathrm{NO}_{4}$ : C 61.64; $\mathrm{H}$ 7.56; N 5.53. Found: C 61.39; H 7.87; N 5.31.

2-(3-Isopropyl-2,5-dioxo-2,5-dihydropyrrol-1-yl)-3-phenylpropionic acid (7d). Oil. Yield 64\% $\mathrm{Rf}=0.6$ (diethyl ether/hexane 50:50). ${ }^{1} \mathrm{H} \mathrm{NMR}\left(300 \mathrm{MHz}, \mathrm{CDCl}_{3}\right): \delta: 7.10-7.30(5 \mathrm{H}, \mathrm{m}), 6.12(1 \mathrm{H}$, s), $4.98(1 \mathrm{H}, \mathrm{dd}, \mathrm{J}=6.3,10.5 \mathrm{~Hz}), 3.40-3.55(2 \mathrm{H}, \mathrm{m}), 2.68-2.80(1 \mathrm{H}, \mathrm{m}), 1.18(3 \mathrm{H}, \mathrm{d}, \mathrm{J}=7.0 \mathrm{~Hz})$, $1.09(3 \mathrm{H}, \mathrm{d}, \mathrm{J}=7.0 \mathrm{~Hz}) .{ }^{13} \mathrm{C} \mathrm{NMR}\left(75 \mathrm{MHz}, \mathrm{CDCl}_{3}\right): \delta: 174.5,170.2,169.9,155.9,136.5,129.0(\mathrm{x}$ 2), 128.7 (x 2), 127.0, 124.5, 52.9, 34.4, 25.8, 20.7. Anal. Calcd for $\mathrm{C}_{16} \mathrm{H}_{17} \mathrm{NO}_{4}$ : C 66.89; H 5.96; N. 4.88. Found: C 66.55; H 6.12; N. 4.65.

\section{ACKNOWLEDGMENT}

The authors gratefully acknowledge the University of Milan (FIRST funds) for financial support.

\section{REFERENCES}

1. Putri, S.P.; Kinoshita, H.; Ihara, F.; Igarashi, Y.; Nihira, T. Farinomalein, a Maleimide-Bearing Compound from the Entomopathogenic Fungus Paecilomyces farinosus.J. Nat. Prod. 2009, 72, 1544-1546.

2. Dorrance, A. E.; Robertson, A. E.; Cianzo, S.; Giesler, I. J.; Gran, C. R.; Draper, M. A.; Tenuta, A. U.; Anderson, T. R. Integrated Management Strategies for Phytophthora sojae Combining Host Resistance and Seed Treatments Plant Disease 2009, 93, 875-882.

3. Miles, W.; Yan, M. Synthesis of farinomalein. Tetrahedron Lett. 2010, 51, 1710-1712.

4. Sharma, V.; Gilbert, T.; Coran, M.; Watanabe, H.. Exploration of the Molecular Origin of the Azinomycin Epoxide: Timing of the Biosynthesis Revealed. Org. Lett. 2008, 10, 4815-4818. 
5. Maury, J; Feray, L.; Bertrand, M. P. Unprecedented Noncatalyzed anti-Carbozincation of Diethyl Acetylenedicarboxylate through Alkylzinc Group Radical Transfer. Org.Lett. 2011, 13, 1884-1887. 6. Akhtar, M.; Botting, N. P.; Cohen, M. A.; Gani, D. Enantiospecific synthesis of 3-substituted aspartic acids via enzymic amination of substituted fumaric acids. Tetrahedron 1987, 43, 58995908.

7. Russell, G.A.; Guo, D.; Baik, W.; Herron, S. J. Electron transfer processes. 44. Alkylations of heterocycles by alkylmercurials. Heterocycles 1989, 28, 143-6.

8. de Figueiredo, R. M.; Oczipka, P.; Frohlich, R.; Christmann, M. Synthesis of 4-Maleimidobutyric Acid and Related Maleimides. Synthesis 2008, 1316-1318.

9. Danelutte, A. P.; Lago, J. H. G.; Young, M. C. M. Kato, M. J. Antifungal flavanones and prenylated hydroquinones fro Piper Crassinervium Kunth. Phytochemistry 2003, 64, 555.

10. Homans, A. L.; Fuchs, A. Direct bioautography on thin-layer chromatograms as a method for detecting fungitoxic substances. J. Chromatogr. 1970, 51, 327. 


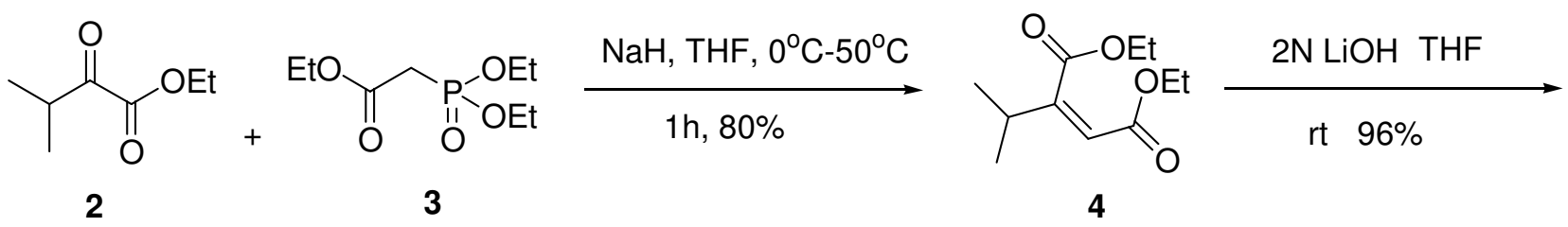<smiles>CC(C)/C(=C/C(=O)O)C(=O)O</smiles>

5
6<smiles>CC(C)C1=CC(=O)N(CCC(=O)O)C1=O</smiles>

1

Scheme 1. Reaction pathway to Farinomalein<smiles>CC(C)C1=CC(=O)OC1=O</smiles><smiles>[R][C@H](N)C(=O)O</smiles>

$\mathrm{AcOH}$, reflux, $1.5 \mathrm{~h}$<smiles>[R]C(C(=O)O)N1C(=O)C=C(C(C)C)C1=O</smiles>

6

7a $\mathrm{R}=\mathrm{H}$

7 b $\mathrm{R}=\mathrm{CH}\left(\mathrm{CH}_{3}\right)_{2}$

7c $\mathrm{R}=\mathrm{CH}_{2} \mathrm{CH}\left(\mathrm{CH}_{3}\right)_{2}$

7d $\mathrm{R}=\mathrm{CH}_{2} \mathrm{Ph}$

Scheme 2. Reaction pathway to Farinomalein analogues 